\title{
Preclinical trial of the multi-targeted lenvatinib in combination with cellular immunotherapy for treatment of renal cell carcinoma
}

\author{
CHENGKUAN CAI $^{1 *}$, JINGYUAN TANG $^{2 *}$, BAIXIN SHEN $^{1}$, LIUCHENG DING $^{1}$, \\ YUNPENG SHAO $^{1}$, ZHENGSEN CHEN $^{1}$, YINCHAO MA ${ }^{1}$, HAOLIANG XUE $^{1}$ and ZHONGQING WEI ${ }^{1}$ \\ ${ }^{1}$ Department of Urology, The Second Affiliated Hospital of Nanjing Medical University, Nanjing, Jiangsu 210011; \\ ${ }^{2}$ Department of Urology, The First Affiliated Hospital of Nanjing Medical University, Nanjing, Jiangsu 210029, P.R. China
}

Received October 9, 2015; Accepted February 7, 2017

DOI: $10.3892 /$ etm.2017.4858

\begin{abstract}
Lenvatinib is an oral, multi-targeted tyrosine kinase inhibitor of vascular endothelial growth factor receptors 1-3, fibroblast growth factor receptors 1-4, platelet-derived growth factor receptor $\beta$, RET and KIT. Cellular immunotherapy has the potential to be a highly targeted treatment, with low toxicity to normal tissues and a high capacity to eradicate tumor tissue. The present study assessed the safety, maximum tolerated dose (MTD) and preliminary antitumor activity of lenvatinib and cellular immunotherapy in a murine model of renal cell carcinoma (RCC). The present study used a therapeutic dose of $0.12 \mathrm{mg}$ lenvatinib and/or $10^{4}$ rat uterine cancer adenocarcinoma ( $\mathrm{RuCa}$ )-sensitized lymphocytes administered once daily continuously in 7-day cycles. Tumor regression was observed in mice with RCC following treatment with lenvatinib and $10^{4} \mathrm{RuCa}$-sensitized lymphocytes. MTD was established as once daily administration of $0.18 \mathrm{mg}$ lenvatinib and $10^{6} \mathrm{RuCa}$-sensitized lymphocytes. The most common treatment-related adverse effects observed were fatigue $(40 \%)$, mucosal inflammation (30\%), proteinuria, diarrhea, vomiting, hypertension and nausea (all 40\%). Combination therapy using lenvatinib and cellular immunotherapy enhanced the antitumor effect induced by single treatments and prolonged the survival of mice with RCC compared with either of the single treatments. Treatment with lenvatinib $(0.12 \mathrm{mg})$ combined with $10^{4}$ RuCa-sensitized lymphocytes was associated with manageable toxicity consistent with individual agents. Further evaluation of this combination therapy in mice with advanced RCC is required. In conclusion, cellular immunotherapy and oncolytic
\end{abstract}

Correspondence to: Dr Zhongqing Wei, Department of Urology, The Second Affiliated Hospital of Nanjing Medical University, 121 Jiangjia Yuan Road, Nanjing, Jiangsu 210011, P.R. China

E-mail: weizhongqing1314@163.com

*Contributed equally

Key words: lenvatinib, cellular immunotherapy, rat uterine cancer adenocarcinoma-sensitized lymphocyte, renal cell carcinoma, antitumor therapy for cancer may be improved by the synergistic effects of lenvatinib and sensitized lymphocytes. In the present study, the inherent antineoplastic and immune stimulatory properties of the two agents were enhanced when used in combination, which may provide a basis for clinical treatment of patients with RCC.

\section{Introduction}

Standard therapies including chemotherapy and radiotherapy for various types of human cancer are toxic and often ineffective (1). Cellular immunotherapy has the potential to be a highly targeted alternative to these therapies, with a high capacity to eradicate tumors and low toxicity to normal tissues (2-4). Cellular immunotherapy often employs active immunization with cells, peptides, proteins or nucleic acids, as well as utilizing adoptive transfer of effector cells that directly target antigens on malignant cells (5). A variety of these approaches have been demonstrated to be successful in treating ovarian and lung cancer $(6,7)$. In the present study, approaches that generate cellular therapies by combining active immunization with cells that use adoptive transfer of effector cells, which directly target RCC cells in xenograft mice, were investigated.

Lenvatinib is an oral, multi-targeted tyrosine kinase inhibitor of vascular endothelial growth factor receptors 1-3 (VEGFR1-3), fibroblast grow th factor receptors 1-4 (FGFR1-4), platelet-derived growth factor receptor $\beta$ (PDGFR- $\beta$ ), RET and KIT. Previous studies have identified VEGF, FGFR1-4, PDGFR- $\beta$, RET and KIT-mediated angiogenesis to be key factors in the development of renal cell carcinoma $(\mathrm{RCC})(8,9)$. Lenvatinib has potent anti-tumor activity against various human tumors in murine xenograft models, mediated by the inhibition of angiogenesis (10). Various agents that inhibit the VEGF pathway have demonstrated clinical benefit in metastatic RCC (11). Lenvatinib, which targets the VEGFR1-3, FGFR1-4, PDGFR- $\beta$, RET and KIT pathways, has also demonstrated clinical benefit in RCC (11). A previous study indicated that lenvatinib combined with everolimus significantly extended overall survival compared with everolimus alone in patients with metastatic RCC (12).

Research suggests that tumors become resistant to therapy via feedback mechanisms that compensate for targeted inhibition (13). The upregulation of hypoxia-inducible factor 1 target genes, such as VEGF, has been implicated in RCC (12). 
Additionally, genetic alterations resulting in the constitutive activation of cellular immunotherapy signaling pathways have been reported to be associated with RCC (14). Therefore, a combination of agents that target VEGF and cellular immunotherapy-mediated pathways may simultaneously block two critical signaling pathways that are activated in RCC, and may overcome an aspect of resistance to single-agent therapy.

$\mathrm{RCC}$, caused by mutations in renal tubular cells, is one of the most common urologic neoplasms (15). Increasing research has focused on the development of comprehensive treatments for RCC $(16,17)$. The present study demonstrated the synergistic effects between cellular immunotherapy and lenvatinib in a murine model of RCC and the landscape of current targeted and cellular immunotherapy for RCC was examined. Our results indicated that cellular immunotherapy combined with targeted agents, such as lenvatinib, is undergoing preclinical trials in RCC. The results of the present study demonstrated that cellular immunotherapy combined with lenvatinib inhibited tumor growth in xenograft mice. Furthermore, it was demonstrated that cellular immunotherapy combined with lenvatinib significantly prolonged the survival of tumor-bearing mice $(\mathrm{P}<0.05)$. These results indicated that cellular immunotherapy combined with lenvatinib treatment may be applied in clinical practice for treatment of patients with RCC.

\section{Materials and methods}

Ethics statement. The present study was conducted in strict accordance with the recommendations in the Guide for the Care and Use of Laboratory Animals of the National Institutes of Health (13). Experimental protocols were approved by the Chinese Association for Laboratory Animal Sciences (Guangzhou, China) and Animal Health Products and the Committee on the Ethics of Animal Experiments Defense Research (Guangzhou, China). All surgery and euthanasia were performed under sodium pentobarbital anesthesia (30 mg/kg, Sunbio Inc., Shanghai, China), and all efforts were made to minimize suffering.

Cell culture and reagents. Cells from a human kidney adenocarcinoma cell line (ACHN), renal carcinoma cell line (786-0) and rat uterine cancer adenocarcinoma $(\mathrm{RuCa})$ cell line were obtained from the National Cancer Institute (NCI) Frederick Cancer Research Facility, the NCI Division of Cancer Treatment Tumor Repository (both Frederick, MD, USA) and American Type Culture Collection (Manassas, VA, USA), respectively. ACHN, 786-0 and RuCa cells were cultured in Dulbecco's modified Eagle's medium supplemented with $10 \%$ heat-inactivated fetal bovine serum (BioWhittaker; Lonza Walkersville, Inc., Walkersville, MD, USA), 3 mM L-glutamine (Meilun Biology Technology Inc., Dalian, China), $50 \mu \mathrm{g} / \mathrm{ml}$ gentamicin (BioWhittaker; Lonza Walkersville, Inc.) and $1 \%$ penicillin/streptomycin. Normal human astrocyte cells (catalogue no. CC-2565; Clonetics ${ }^{\mathrm{TM}}$; Lonza) were purchased from Lonza Walkersville, Inc., and maintained in an AGM Astrocyte Growth Bullet kit (Lonza Walkersville, Inc.), according to the manufacturer's instructions.

Reverse transcription-quantitative polymerase chain reaction (RT-qPCR). Total cellular RNA was extracted from cells using an RNeasy mini kit (Qiagen, Inc., Valencia, CA, USA), according to the manufacturer's instructions. cDNA (10 ng) was used for qPCR with the SYBR-Green Master Mix system (Bio-Rad Laboratories, Inc., Hercules, CA, USA). RNA samples $(1 \mu \mathrm{l})$ were reverse transcribed using the iScript cDNA synthesis kit (catalogue no. 1708890; Bio-Rad Laboratories, Inc., Hercules, CA, USA), according to the manufacturer's instructions. RNA enzyme-free ultrapure water (dilution, 1:10) and the absorbency at wavelengths of 260 and $280 \mathrm{~nm}$ were recorded using an ultraviolet spectrophotometer (UV-1100; Shanghai Mapada Instruments Co., Ltd., Shanghai, China) to determine purity and concentration. The cDNA sample $(1 \mu \mathrm{l})$ was subjected to a PCR reaction, performed in an iCycler thermal cycler using iQ SYBR Green Supermix (both Bio-Rad Laboratories, Inc.). The Fast Real-Time PCR system was used with the following protocol: $25^{\circ} \mathrm{C}$ for $5 \mathrm{~min}, 42^{\circ} \mathrm{C}$ for $1 \mathrm{~h}$ and $70^{\circ} \mathrm{C}$ for $5 \mathrm{~min}$ for a total of 35 cycles. GAPDH levels were measured as an internal control. The forward and reverse primers are as follows: VEGFR, forward 5'-GCCCCGCCT TATGATTCTCTGC-3' and reverse 5'-CTCGCCGCCTCC GTACATGTC-3', PDGFR, forward 5'-CAGCAGTATGAA AGCGTGG-3' and reverse 5'-GGAAGAAAAGGTTGGCAG AG-3', RET, forward 5'-GCAGACGACGGGTCAGAT-3' and reverse 5'-GACTGACCCGTCGTGACG-3' and KIT, forward 5'-TGGCAAGACAACGTGAAAGA-3' and reverse 5'-AAC TGGGAAAATGCATCTGG-3'. Primers were synthesized by Invitrogen (Thermo Fisher Scientific, Inc., Waltham, MA, USA). The expression level of mRNA was denoted as mRNA/GAPDH, assessed using the $2^{-\Delta \Delta \mathrm{Cq}}$ protocol, following detection of expression levels in all three cell types (18). PCR was repeated three times in triplicate.

MTT cytotoxicity assays. ACHN, 786-0 and RuCa cells $\left(5 \times 10^{5} / \mathrm{ml}\right)$ were cultured in 96 well plates for $24 \mathrm{~h}$ at $5 \% \mathrm{CO}_{2}$ and $37^{\circ} \mathrm{C}$ and incubated with lenvatinib $(0.1 \mathrm{mg} / \mathrm{ml}$; Sunbio Inc.) and/or sensitized lymphocytes (1x10 $/ \mathrm{ml}$; Sunbio Inc.) for 48, 72 and $96 \mathrm{~h}$ at $37^{\circ} \mathrm{C}$ in triplicate for each condition. Phosphate buffered saline (PBS) was added instead of the lenvatinib or sensitized lymphocytes to act as a control. At each time point, $20 \mu \mathrm{l}$ MTT reagent $(5 \mathrm{mg} / \mathrm{ml})$ in PBS solution was added to each well and the plates were incubated at $37^{\circ} \mathrm{C}$ for $4 \mathrm{~h}$. The majority of the medium was removed and $100 \mu 1$ dimethyl sulfoxide was added into the wells to solubilize the formazan crystals. The optical density was measured using a microplate reader (Bio-Rad Laboratories, Inc.) at a wavelength of $450 \mathrm{~nm}$.

Induction of apoptosis in RCC cells. ACHN, 786-0 and RuCa cells were grown at $37^{\circ} \mathrm{C}$ in $5 \% \mathrm{CO}_{2}$ until $80 \%$ confluence was reached. Apoptosis was assessed by incubation of these cells with lenvatinib $(0.1 \mathrm{mg} / \mathrm{ml})$ and/or sensitized lymphocytes $\left(1 \times 10^{5} / \mathrm{ml}\right)$ for $72 \mathrm{~h}$ at $5 \% \mathrm{CO}_{2}$ and $37^{\circ} \mathrm{C}$. Following incubation with the lenvatinib and/or sensitized lymphocytes, the cells were trypsinized and collected. Subsequently, the cells were washed twice for $30 \mathrm{~min}$ in cold PBS, adjusted to $1 \times 10^{6}$ cells/ml with PBS and labeled with annexin-V-fluorescein isothiocyanate (FITC) and propidium idodide (PI) using an annexin-V-FITC kit (BD Biosciences, San Jose, CA, USA). Apoptotic cells were quantified using a FACScan flow cytometer (BD Accuri ${ }^{\text {TM }}$ C6 Plus; BD Biosciences, San Jose, CA, USA). 
Purification of blood derived cells and cytotoxicity assay. Murine peripheral blood mononuclear cells (PBMC) were isolated from the buffy coat of 40 healthy male mice purchased from the Animal Center of Nanjing Medical University (Nanjing, China) between 8-9 weeks of age with a body weight of 20-25 g by Ficoll-Paque (GE Healthcare Life Sciences, Chalfont, UK) density gradient centrifugation. Mice were maintained in a room with constant temperature $\left(22 \pm 1^{\circ} \mathrm{C}\right)$ and a 12 -h light/dark cycle and housed in cages ( $<5$ mice per cage) with ad libitum access to food and water. $\mathrm{T}$ cells were purified from PBMCs via magnetic bead separation using antibody-coated Dynabeads (Invitrogen; Thermo Fisher Scientific, Inc.) and a Dynal T cell negative isolation kit (Invitrogen; Thermo Fisher Scientific, Inc.) containing monoclonal antibodies against cluster of differentiation (CD) 14, CD16, CD56, human leukocyte antigen class II antigen D related (HLA-DR)/DP and beads coated with an Fc-specific human immunoglobulin (Ig) G4 against mouse IgG, or using anti-CD3 MACSmicrobeads (Miltenyi Biotec $\mathrm{GmbH}$, Bergisch Gladbach, Germany), according to the manufacturer's guidelines. The purified T cells were stimulated at $37^{\circ} \mathrm{C}$ for $72 \mathrm{~h}$ with ACHN, 786-0 or RuCa cells. All cells were recovered from the culture, counted and used as effector cells.

Tumor cells $\left(2 \times 10^{6}\right)$ were labeled with $200 \mu \mathrm{Ci}$ chromium 51 (Zen-bio Inc., Shanghai, China; ${ }^{51} \mathrm{Cr}$ ) for $1 \mathrm{~h}$ at $37^{\circ} \mathrm{C}$, washed four times with PBS and resuspended at $2 \times 10^{5} \mathrm{cell} / \mathrm{s} / \mathrm{ml}$ in PBS. To quantify T-cell induced cell death,tumorcells $\left(10^{4} \mathrm{cells} / \mathrm{well}\right)$ were incubated at $37^{\circ} \mathrm{C}$ with varying numbers of T cells $\left(10^{3}\right.$, $10^{4}, 10^{5}, 10^{6}$ and $10^{7}$ ) for $48 \mathrm{~h}$. The assay was performed in96-well round-bottom plates in triplicate, and the percentage of specific lysis was calculated after analysis of cytotoxicity in the supernatants of the wells using the following formula: $100 \times$ [experimental counts per minute (cpm)-spontaneous $\mathrm{cpm}) /($ total $\mathrm{cpm}$-spontaneous cpm)]. Spontaneous and total ${ }^{51} \mathrm{Cr}$ release values were determined in the presence of either PBS alone or 10\% Triton X-100. RuCa-sensitized lymphocytes were used in animal experiments.

Enzyme-linked immunosorbent assay (ELISA). The production of interferon- $\gamma$ (IFN- $\gamma)$ from the cells was analyzed using an ELISA assay. ELISA was performed using an sandwich ELISA kit (catalogue no. ab193969; Abcam, Cambridge, UK) to determine the IFN- $\gamma$ protein expression level in the mice spleen, obtained during surgery following sacrifice. Briefly, the spleen was homogenized in $1 \mathrm{ml}$ of PBS containing 1\% Triton $\mathrm{X}-100$ using the plunger of a 10-ml syringe, and centrifuged at $7155 \mathrm{x}$ g for $5 \mathrm{~min}$ at $4^{\circ} \mathrm{C}$, and the total protein concentration of the supernatant was determined using a bicinchoninic acid assay as previously described (19). IFN- $\gamma$ antibody (catalogue no. ab175878; Abcam, Cambridge, UK), horseradish peroxidase-streptavidin and TMB One-Step Substrate (Abcam) reagent were incubated in turn and in 96-well plates at room temperature according to the manufacturer's protocol. Stop solution (Abcam) was then added and the optical density (OD) was read on a microplate reader at $450 \mathrm{~nm}$ (Thermo Fisher Scientific, Inc.).

Animal experiments. A total of 40 male BALC/c mice (mean age of 24 months and a body weight of 20-25 g) with RCC were purchased from the West China Experimental Animal Center of Sichuan University (Sichuan, China). Mice were maintained in a room with constant temperature $\left(22 \pm 1^{\circ} \mathrm{C}\right)$ and a 12-h light/dark cycle, and cages in groups $<5$ per cage with ad libitum access to food and water. The mice were split into 4 groups, containing 10 mice. Each mouse in the treatment group received $0.12 \mathrm{mg}$ lenvatinib or/and $10^{4} \mathrm{RuCa}$-sensitized lymphocytes administered once daily continuously in 7-day cycles. The mice in the control group received $2 \mathrm{ml}$ PBS. Tumor dimensions were measured 10 times, every 2 days. Tumor volumes were calculated according to the following formula: Length $\mathrm{x}$ width ${ }^{2} \mathrm{x} 0.52$. On day 30 after the final inoculation, tumor tissues were extracted and analyzed using RT-qPCR assays (as described). Mice were kept alive for 180 days to assess the effects of the treatments on survival.

Efficacy safety assessment. Efficacy assessment included the maximum toxicity dose in mice models and the dose-limiting toxicity in the presence and absence of cellular immunotherapy and lenvatinib. Safety assessments included the incidence rates $(>10 \%)$ of the most frequent treatment-emergent adverse events in the 30-day treatment period.

Statistical analysis. All data are presented as the mean \pm standard deviation. Analysis was performed using SPSS software version 20.0 (IBM Corp, Armonk, NY, USA). Statistical significance of differences between mean values was assessed using Student's t-test for unpaired data. Comparisons of data between multiple groups were performed with one-way analysis of variance, followed by Newman Keuls post hoc test. $\mathrm{P}<0.05$ was considered to indicate a statistically significant difference.

\section{Results}

Antineoplastic evaluation of lenvatinib and sensitized lymphocytes in vitro. In order to investigate the effects of cellular immunotherapy and lenvatinib on renal cancer cells, RuCa, ACHN and 786-0 cells were treated with $0.12 \mathrm{mg}$ lenvatinib or/and $10^{4}$ sensitized lymphocytes. The results in Fig. 1 Demonstrated that the cytotoxicity of RuCa, ACHN and 786-0 cells when treated with lenvatinib or/and sensitized lymphocyte increased in a time dependent manner after 48,72 and $96 \mathrm{~h}$ of treatment. The results of the MTT assay indicated that the growth of $\mathrm{RuCa}, \mathrm{ACHN}$ and 786-0 cells were inhibited after treatment with sensitized lymphocytes and/or lenvatinib (Fig. 1).

Duration of treatment, dose-limiting toxicities and maximum tolerated dose (MTD). Median overall duration of treatment was 7 days across all dosing cohorts. The dosing cohorts included $0.08,0.12,0.16,0.18,0.20$ and $0.24 \mathrm{mg}$ of lenvatinib and $10^{3}, 10^{4}, 10^{5}, 10^{6}$ and $10^{7}$ of sensitized lymphocytes. In our results, $0.18 \mathrm{mg}$ lenvatinib once daily and $10^{6}$ sensitized lymphocytes once daily was identified as the MTD.

Mice received at least one dose of $0.18 \mathrm{mg}$ lenvatinib and $10^{6}$ with post baseline safety evaluation. Following the last lenvatinib and sensitized lymphocyte dose, the most common treatment-related adverse events (AEs) were hypertension, diarrhea, constipation, weight loss, loss of appetite, proteinuria and rash (Table I). The most common treatment-related AEs with a toxicity criteria grade $\geq 3$ were hypertriglyceridemia, proteinuria, diarrhea and fatigue (10\% each; Table II). 

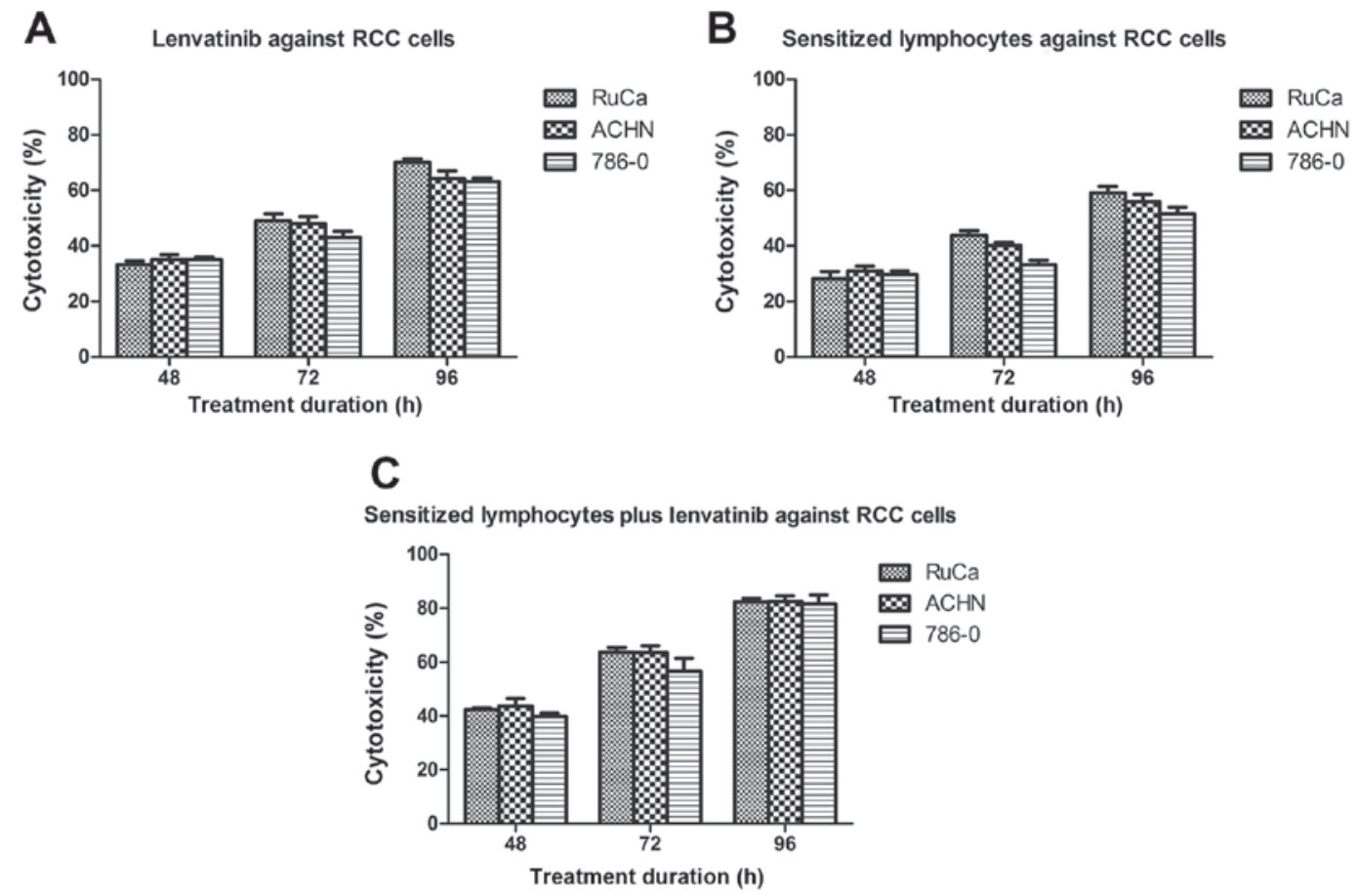

Figure 1. Lenvatinib or/and sensitized lymphocytes induce different degrees of necrosis of RCC cells according to MTT assays. Necrosis of RCC cells treated with (A) lenvatinib, (B) sensitized lymphocytes, (C) lenvatinib plus sensitized lymphocytev. Data are presented as the mean \pm standard deviation. RCC, renal cell carcinoma; $\mathrm{RuCa}$, rat uterine cancer adenocarcinoma; $\mathrm{ACHNv}$, human kidney adenocarcinoma cell line; 786-0, renal carcinoma cell line.
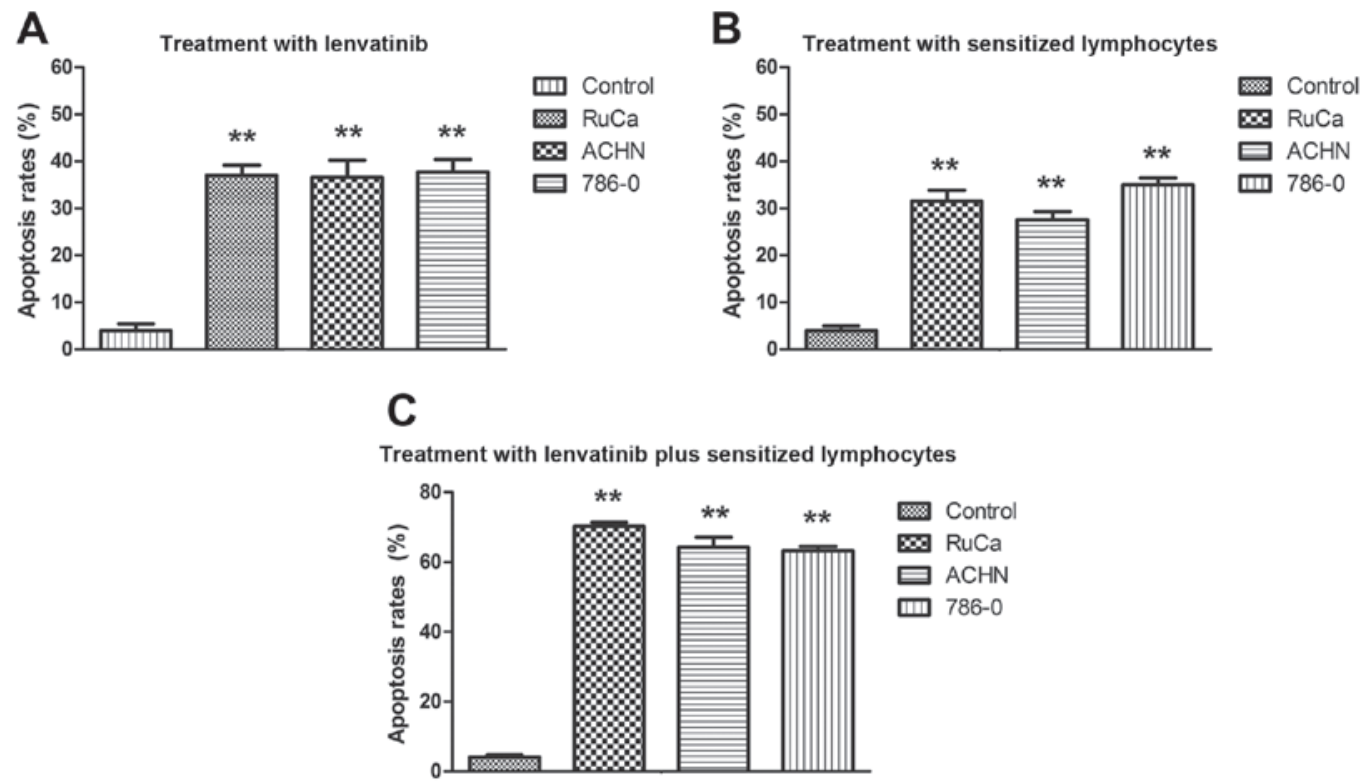

Figure 2. Treatment with lenvatinib and sensitized lymphocytes induces apoptosis in renal cell carcinoma. Apoptosis of ACHN, 786-0 and RuCa cells induced by treatment with (A) lenvatinib, (B) sensitized lymphocytes and (C) lenvatinib plus sensitized lymphocytes. Values are presented as the mean \pm standard deviation of triplicate samples. One-way analysis of variance revealed significant differences. ${ }^{* *} \mathrm{P}<0.01 \mathrm{vs}$. the control. RuCa, rat uterine cancer adenocarcinoma; ACHN, human kidney adenocarcinoma cell line; 786-0, renal carcinoma cell line.

Effect of cellular immunotherapy and lenvatinib treatment on apoptosis of RCC cells. The morphological changes induced by cellular immunotherapy and lenvatinib treatment in $\mathrm{RuCa}, \mathrm{ACHN}$ and 786-0 cells indicated that treatment with sensitized lymphocytes and lenvatinib inhibited the growth of renal tumors. Therefore, the therapeutic effects of sensitized lymphocytes and lenvatinib in vitro on human and mouse RCC cells were investigated. Results demonstrated that apoptosis of ACHN, 786-0 and RuCa cells significantly increased after treatment with lenvatinib compared with the control group, and the apoptosis of ACHN, 786-0 and RuCa cells significantly increased after treatment with combined lenvatinib and sensitized lymphocyte treatment group compared with the lenvatinib and sensitized lymphocyte groups $(\mathrm{P}<0.01$; Fig. 2). The mRNA expression levels of several genes associated with lenvatinib-mediated apoptosis, including VEGFR, 
Table I. Treatment-related adverse events with an overall incidence $\geq 10 \%$.

\begin{tabular}{|c|c|c|c|c|}
\hline \multirow[b]{2}{*}{ Adverse event } & \multirow[b]{2}{*}{ Total $(n=54)$} & \multicolumn{3}{|c|}{ Treatment } \\
\hline & & $\begin{array}{l}\text { Lenvatinib } 0.10 \mathrm{mg} \\
+\mathrm{SLYS} 10^{3}(\mathrm{n}=18)\end{array}$ & $\begin{array}{l}\text { Lenvatinib } 0.12 \mathrm{mg} \\
+\mathrm{SLYS} 10^{4}(\mathrm{n}=18)\end{array}$ & $\begin{array}{l}\text { Lenvatinib } 0.14 \mathrm{mg} \\
+\mathrm{SLYS} 10^{5}(\mathrm{n}=18)\end{array}$ \\
\hline Hypertension & 8 & 2 & 3 & 3 \\
\hline Diarrhea & 8 & 3 & 4 & 1 \\
\hline Proteinuria & 10 & 2 & 4 & 4 \\
\hline Vomiting & 4 & 2 & 2 & 0 \\
\hline Lethargy & 3 & 0 & 1 & 2 \\
\hline Rash & 7 & 2 & 4 & 1 \\
\hline Fatigue & 5 & 1 & 2 & 3 \\
\hline Constipation & 10 & 2 & 3 & 4 \\
\hline Weight decrease & 8 & 3 & 1 & 4 \\
\hline Decreased appetite & 8 & 3 & 2 & 3 \\
\hline Epistaxis & 3 & 0 & 1 & 2 \\
\hline Hypertriglyceridemia & 3 & 1 & 1 & 1 \\
\hline Edema peripheral & 3 & 1 & 1 & 1 \\
\hline
\end{tabular}

SLYS, sensitized lymphocytes.

Table II. Treatment-related hypertension and proteinuria according to common toxicity criteria grade.

\begin{tabular}{|c|c|c|c|c|}
\hline \multirow[b]{2}{*}{ Adverse event } & \multirow[b]{2}{*}{ Total $(n=36)$} & \multicolumn{3}{|c|}{ Treatment } \\
\hline & & $\begin{array}{l}\text { Lenvatinib 0.10mg } \\
+\operatorname{SLYS~} 10^{3}(\mathrm{n}=12)\end{array}$ & $\begin{array}{l}\text { Lenvatinib } 0.12 \mathrm{mg} \\
+\mathrm{SLYS} 10^{4}(\mathrm{n}=12)\end{array}$ & $\begin{array}{l}\text { Lenvatinib } 0.14 \mathrm{mg} \\
+\operatorname{SLYS~} 10^{5}(\mathrm{n}=12)\end{array}$ \\
\hline Hypertriglyceridemia & 16 & 4 & 5 & 7 \\
\hline Grade 1 & 4 & 1 & 1 & 2 \\
\hline Grade 2 & 9 & 2 & 3 & 4 \\
\hline Grade 3 & 3 & 1 & 1 & 1 \\
\hline Proteinuria & 20 & 2 & 6 & 12 \\
\hline Grade 1 & 4 & 0 & 1 & 3 \\
\hline Grade 2 & 10 & 2 & 3 & 5 \\
\hline Grade 3 & 6 & 0 & 2 & 4 \\
\hline Fatigue & 11 & 2 & 3 & 6 \\
\hline Grade 1 & 3 & 0 & 1 & 2 \\
\hline Grade 2 & 6 & 1 & 2 & 3 \\
\hline Grade 3 & 2 & 0 & 0 & 2 \\
\hline Diarrhea & 18 & 3 & 5 & 10 \\
\hline Grade 1 & 4 & 1 & 1 & 2 \\
\hline Grade 2 & 8 & 2 & 2 & 4 \\
\hline Grade 3 & 4 & 0 & 1 & 3 \\
\hline
\end{tabular}

SLYS, sensitized lymphocytes.

PDGFR, RET and KIT, were analyzed using RT-qPCR. As demonstrated in Fig. 3, mRNA expression levels of VEGFR, PDGFR, RET and KIT were significantly decreased in RCC cells following treatment with lenvatinib compared with the control and they also were significantly decreased in RCC cells following treatment with combined lenvatinib and sensitized lymphocyte treatment group compared with lenvatinib group $(\mathrm{P}<0.01)$. These results suggest that lenvatinib inhibited the expression of angiogenesis-related genes.

Effect of cellular immunotherapy and lenvatinib treatment of RCC on survival in vivo. To explore whether cellular 

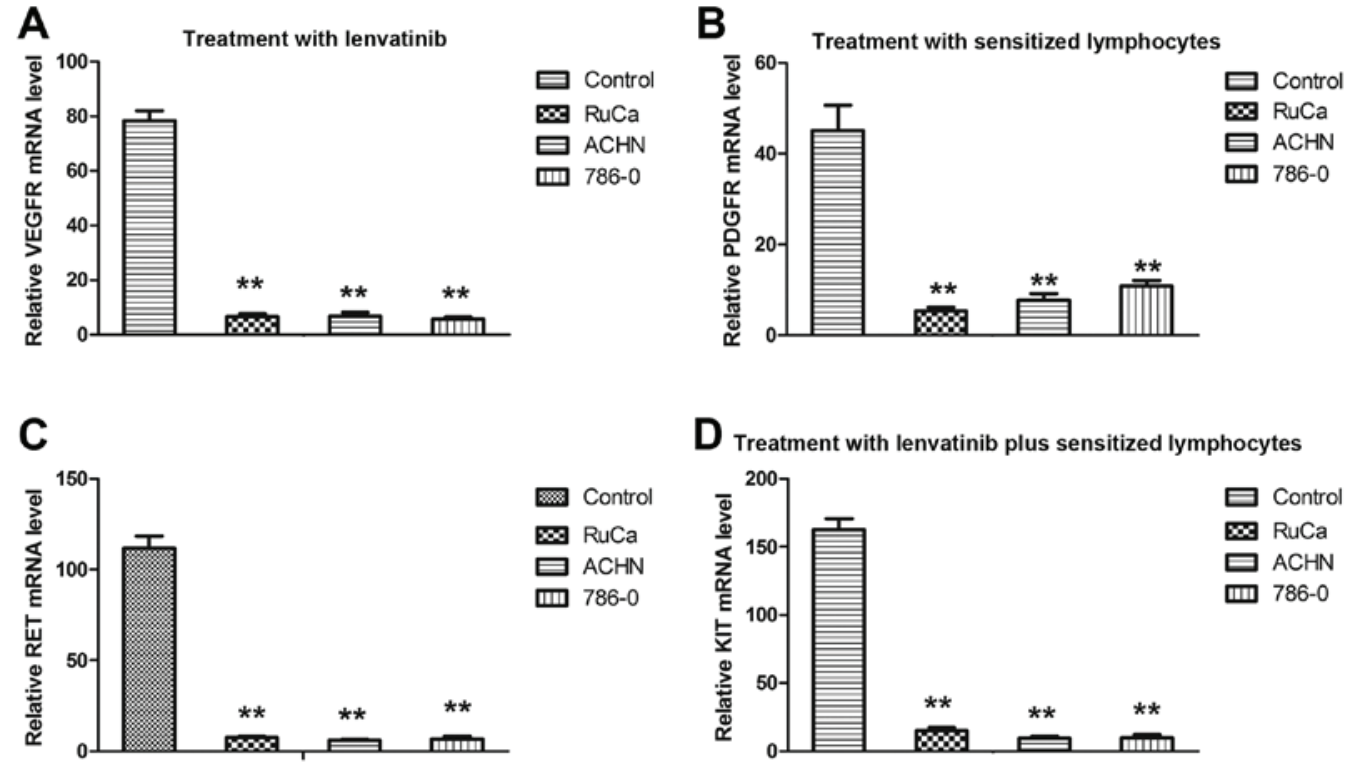

D Treatment with lenvatinib plus sensitized lymphocytes

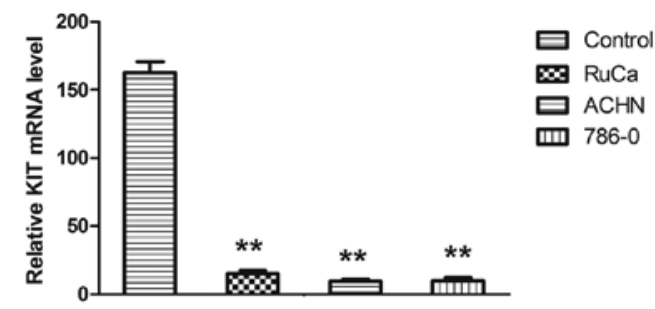

Figure 3. Treatment with lenvatinib and sensitized lymphocytes reduces apoptosis-related gene expression levels. (A) Effect of lenvatinib treatment on VEGFR mRNA expression level. (B) Effect of treatment with sensitized lymphocytes on PDGFR mRNA expression level. (C) Effect of lenvatinib treatment on RET mRNA expression level. (D) Effect of treatment with lenvatinib plus sensitized lymphocytes on KIT mRNA expression level. Expression of each gene was calculated relative to the expression of $\beta$-actin and the results are expressed as the $n$-fold difference relative to $\beta$-actin. Data are presented as the mean \pm standard deviation of triplicate samples. One-way analysis of variance revealed significant differences. ${ }^{* *} \mathrm{P}<0.01$ vs. the control. VEGFR, vascular endothelial growth factor receptor; PDGFR, platelet-derived growth factor receptor; RuCa, rat uterine cancer adenocarcinoma; ACHN, human kidney adenocarcinoma cell line; 786-0, renal carcinoma cell line.
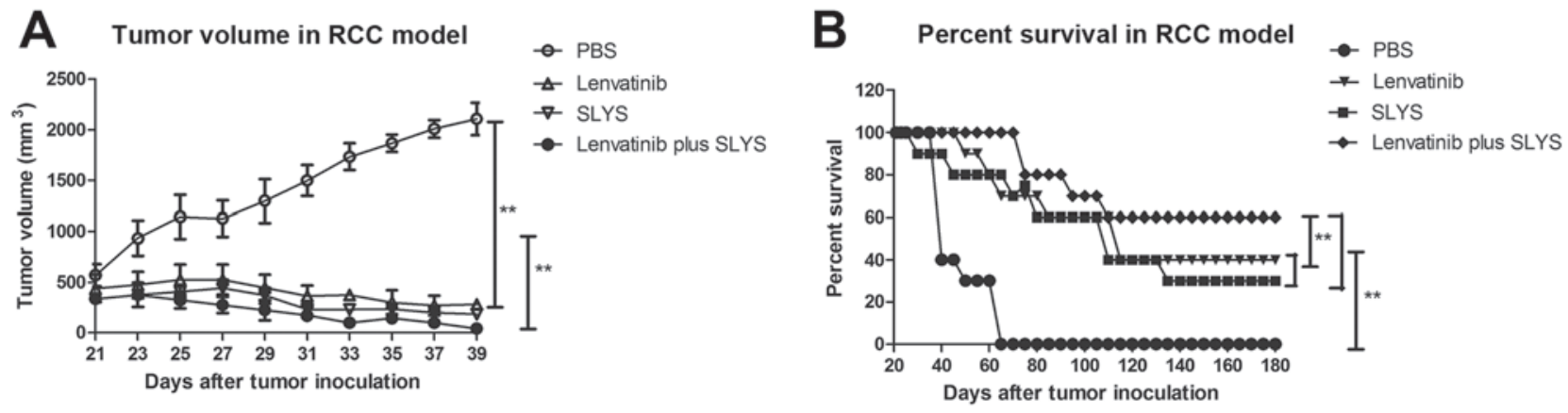

Figure 4. Treatment with lenvatinib plus SLYS effectively suppresses tumor growth and prolongs survival in mice with RCC. Effect of lenvatinib and SLYS on (A) tumor volume and (B) survival. Data are presented as the mean \pm standard deviation of triplicate samples. One-way analysis of variance revealed a significant effect. ${ }^{* *} \mathrm{P}<0.01$ vs. the PBS control. PBS, phosphate buffered saline; SLYS, sensitized lymphocytes; RCC, renal cell carcinoma.

immunotherapy and lenvatinib are effective anticancer agents in vivo, the anti-tumor activity of the sensitized lymphocytes and lenvatinib in a murine model of RCC was investigated. Results demonstrated that the tumor volume in mice treated with sensitized lymphocytes plus lenvatinib was significantly lower than that of the mice treated with PBS or a single agent $(\mathrm{P}<0.01$; Fig. 4A). Furthermore, long-term survival was assessed for 180 days after treatment with cellular immunotherapy and lenvatinib treatment. As demonstrated in Fig. 4B, cellular immunotherapy and lenvatinib $(n=10$ in each group) significantly prolonged the survival of mice compared with the control group $(\mathrm{P}<0.01)$. Furthermore, the combined treatment of cellular immunotherapy and lenvatinib was significantly more effective at prolonging survival of the mice than either of the treatments alone $(\mathrm{P}<0.01$; Fig. 4B). These results indicate that combined treatment of sensitized lymphocytes plus lenvatinib is strong enough to partially protect the animals and eliminate the tumor cells, which translated into long-term survival. The maximum percent tumor change from baseline to post baseline nadir is demonstrated by the ELISA results presented in Fig. 5 and illustrates the immune responses of the mice to combined therapy.

\section{Discussion}

Therapeutic targeting of VEGF-mediated pathways has increased the number of available treatment options for human cancer $(10,20,21)$. A number of challenges remain to allow the treatment to overcome the resistance to single-agent therapies (22-24). Combinations of anticancer agents have previously been demonstrated to have strong therapeutic effects (25-27). Optimal treatment schemes using these agents have been 
A

CTL response against RuCa cells

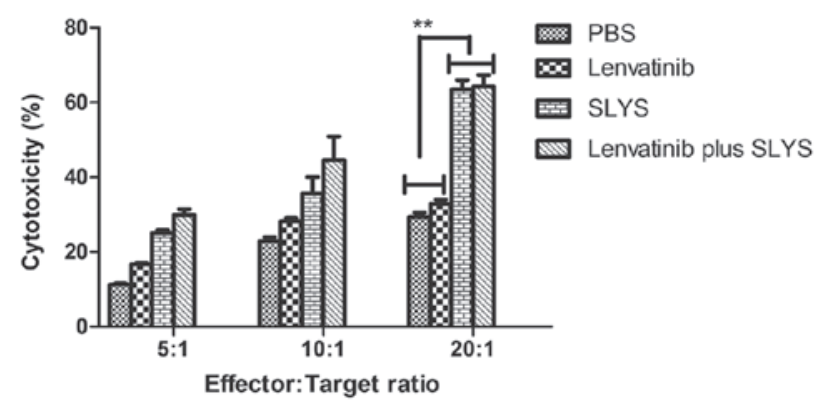

B RuCa-specific splenocyte IFN-y release

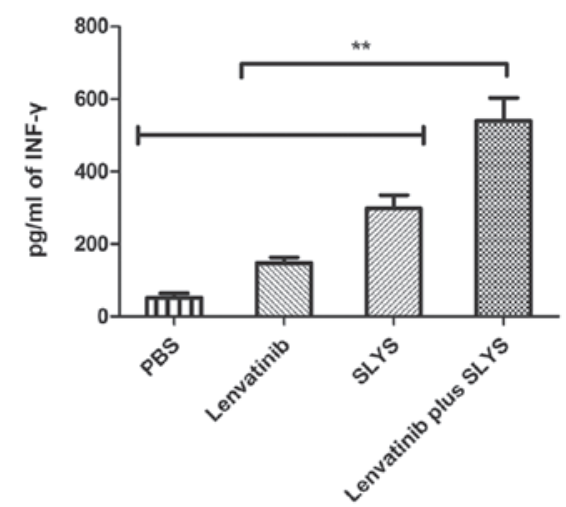

Figure 5. Treatment with lenvatinib plus SLYS enhances immune responses against tumor cells in vivo. (A) CTL response against renal cell carcinoma. (B) Tumor-specific IFN- $\gamma$ released from stimulated splenocytes in a murine model of renal cell carcinoma. Data are presented as the mean \pm standard deviation of triplicate samples. One-way analysis of variance revealed a significant effect. ${ }^{* *} \mathrm{P}<0.01$ vs. the PBS control. PBS, phosphate buffered saline; SLYS, sensitized lymphocytes; CTL, cytotoxic T lymphocyte; IFN, interferon.

defined and clinical development of combinations of anticancer agents have demonstrated low toxicity (28). The most evident and efficient effects are induced by cellular immunotherapy and targeted therapy (28). However, there is a continued requirement for more effective combinations of targeted agents and cellular immunotherapies that demonstrate improved efficacy, are manageable and have low toxicity (29-31).

A previous study demonstrated that the MTD and recommended phase II dose in this phase I experiment was confirmed to be $18 \mathrm{mg}$ lenvatinib in combination with $5 \mathrm{mg}$ everolimus once daily for treatment of RCC (12). In the present study, cellular immunotherapy and targeted therapy was introduced to treat RCC in a xenograft murine model. At the MTD and lower-dose cohort, the combination of lenvatinib and cellular immunotherapy was associated with manageable toxicity. Treatment-related AEs were consistent with class effects typical of VEGFR-targeted therapy and immunotherapy (32). A previous study indicates that fatigue, mucosal inflammation, proteinuria, hypertension, as well as gastrointestinal toxicity, occurred at a low frequency (32). Another previous study indicated that proteinuria, hypertriglyceridemia, diarrhea, and fatigue were the most common treatment-related TEAEs (33).

A phase I study of lenvatinib, which is a multi-targeted tyrosine kinase inhibitor, in patients with advanced solid tumors demonstrated that lenvatinib is an efficient anticancer agent (34); however, only $9 \%$ of patients had a partial response to treatment and so the results of the study suggested that lenvatinib as a monotherapy had limited efficacy for cancer patients. A previous study by Yamada et al (35) reported that lenvatinib has manageable toxicity when administered in a 2-week-on/1-week-off cycle and demonstrated preliminary activity for durable disease control. However, result analysis suggested that antiangiogenic activity correlated with antitumor activity in patients with partial therapeutic effects (35). In a phase II trial combining the treatment doses of bevacizumab and everolimus, median progression-free survival in previously treated patients was 7.1 months (23\%) (36). Therefore, the combination regimen was associated with a high cure rate compared with single agent treatment $(37,38)$. The responses observed with these combination therapies confer an advantage over single-agent therapy; however, further study on the use of these combination therapies is required (39).

In the present exploratory analysis of tumor therapy, treatment with lenvatinib $(0.12 \mathrm{mg})$ and $10^{4} \mathrm{RuCa}$-sensitized lymphocytes once daily resulted in a mean survival rate of $80 \%$, and median progression-free survival of $\sim 180$ days in mice with RCC. In conclusion, the present study used two anticancer agents, lenvatinib $(0.12 \mathrm{mg})$ combined with $10^{4}$ RuCa-sensitized lymphocytes, once daily to treat RCC in mice. The majority of treatment-related AEs were consistent with positive effects of immunotherapy and multi-targeted tyrosine kinase inhibitors, and were managed effectively by administration of lenvatinib and sensitized lymphocytes continuously in 7-day cycles. Notably, beneficial effects of cellular immunotherapy and targeted therapy may further elucidate the clinical value of combination therapy for cancer treatment.

\section{Acknowledgements}

The present study was supported by the National Natural Science Funding of China (grant nos. 81570687, 81300572 and 81500758).

\section{References}

1. Hui D, Elsayem A, Li Z, De La Cruz M, Palmer JL and Bruera E: Antineoplastic therapy use in patients with advanced cancer admitted to an acute palliative care unit at a comprehensive cancer center: A simultaneous care model. Cancer 116: 2036-2043, 2010.

2. Lage A: Immunotherapy and complexity: Overcoming barriers to control of advanced cancer. MEDICC Rev 16: 65-72, 2014.

3. Massarelli E, Papadimitrakopoulou V, Welsh J, Tang C and Tsao AS: Immunotherapy in lung cancer. Transl Lung Cancer Res 3: 53-63, 2014.

4. No authors listed: 'Immunotherapy of metastatic breast cancer patients with vitamin D-binding protein-derived macrophage activating factor (GcMAF)' by Yamamoto, N., Suyama, H., Yamamoto, N. and Ushijima, N. Int J Cancer 135: 1509, 2014.

5. Jackson CM, Lim M and Drake CG: Immunotherapy for brain cancer: Recent progress and future promise. Clin Cancer Res 20: 3651-3659, 2014.

6. Mostafa AA and Morris DG: Immunotherapy for lung cancer: Has it finally arrived? Front Oncol 4: 288, 2014. 
7. Wen Y, Graybill WS, Previs RA, Hu W, Ivan C, Mangala LS, Zand B, Nick AM, Jennings NB, Dalton HJ, et al: Immunotherapy targeting folate receptor induces cell death associated with autophagy in ovarian cancer. Clin Cancer Res 21: 448-459, 2015.

8. Rini BI and Atkins MB: Resistance to targeted therapy in renal-cell carcinoma. Lancet Oncol 10: 992-1000, 2009.

9. Eichelberg C, Junker K, Ljungberg B and Moch H: Diagnostic and prognostic molecular markers for renal cell carcinoma: A critical appraisal of the current state of research and clinical applicability. Eur Urol 55: 851-863, 2009.

10. Hutson TE: Targeted therapies for the treatment of metastatic renal cell carcinoma: Clinical evidence. Oncologist 16 (Suppl 2): S14-S22, 2011.

11. Kuznar W: Lenvatinib extends survival in metastatic renal-cell carcinoma. Am Health Drug Benefits 8: 18, 2015.

12. Molina AM, Hutson TE, Larkin J, Gold AM, Wood K, Carter D, Motzer R and Michaelson MD: A phase $1 \mathrm{~b}$ clinical trial of the multi-targeted tyrosine kinase inhibitor lenvatinib (E7080) in combination with everolimus for treatment of metastatic renal cell carcinoma (RCC). Cancer Chemother Pharmacol 73: 181-189, 2014.

13. Shameem R, Hamid MS, Xu KY and Wu S: Comparative analysis of the effectiveness of abiraterone before and after docetaxel in patients with metastatic castration-resistant prostate cancer. World J Clin Oncol 6: 64-72, 2015.

14. Hutchinson L: Immunotherapy. Significant overall survival advantage for RCC patients treated with autologous tumor lysate vaccine. Nat Rev Clin Oncol 7: 300, 2010.

15. Han MH, Park C, Kwon TK, Kim GY, Kim WJ, Hong SH, Yoo YH and Choi YH: The histone deacetylase inhibitor trichostatin A sensitizes human renal carcinoma cells to TRAIL-induced apoptosis through down-regulation of c-FLIPL. Biomol Ther (Seoul) 23: 31-38, 2015

16. Iacovelli R, Alesini D, Palazzo A, Trenta P, Santoni M, De Marchis L, Cascinu S, Naso G and Cortesi E: Targeted therapies and complete responses in first line treatment of metastatic renal cell carcinoma. A meta-analysis of published trials. Cancer Treat Rev 40: 271-275, 2014

17. Brugarolas J: Renal-cell carcinoma-molecular pathways and therapies. N Engl J Med 356: 185-187, 2007.

18. Livak KJ and Schmittgen TD: Analysis of relative gene expression data using real-time quantitative PCR and the 2(-Delta Delta C(T)) Method. Methods 25: 402-408, 2001

19. Weiser JR, Ricapito NG, Yueh A, Weiser EL and Putnam D: A mechanistic analysis of the quantitation of $\alpha$-hydroxy ketones by the bicinchoninic acid assay. Anal Biochem 430: 116-122, 2012.

20. Villanueva MT: Targeted therapies: Congratulations, you are still in the running towards becoming ovarian-next-top treatment. Nat Rev Clin Oncol 8: 570, 2011.

21. Ngeow J, Tan IB and Choo SP: Targeted therapies in the treatment of gastric cancer. Asia Pac J Clin Oncol 7: 224-235, 2011

22. Sacco PC, Maione P, Rossi A, Bareschino MA, Schettino C Guida C, Elmo M, Ambrosio R, Barbato V, Zeppa R, et al: Combination of radiotherapy and targeted therapies in the treatment of locally advanced non-small cell lung cancer. Target Oncol 6: 171-180, 2011.

23. Batist G, Wu JH, Spatz A, Miller WH Jr, Cocolakis E, Rousseau C, Diaz Z, Ferrario C and Basik M: Resistance to cancer treatment: The role of somatic genetic events and the challenges for targeted therapies. Front Pharmacol 2: 59, 2011.

24. Aggarwal R and Ryan CJ: Castration-resistant prostate cancer: Targeted therapies and individualized treatment. Oncologist 16: 264-275, 2011
25. Rivera F, López-Tarruella S, Vega-Villegas ME and Salcedo M Treatment of advanced pancreatic cancer: From gemcitabine single agent to combinations and targeted therapy. Cancer Treat Rev 35: 335-339, 2009.

26. Bunn PA Jr and Kelly K: Combinations of three chemotherapeutic agents and two chemotherapeutic agents plus a targeted biologic agent in the treatment of advanced non small-cell lung cancer. Clin Lung Cancer 2 (Suppl 1): S23-S28, 2000.

27. Cabrespine A, Bay JO, Barthomeuf C, Curé H, Chollet $\mathrm{P}$ and Debiton E: In vitro assessment of cytotoxic agent combinations for hormone-refractory prostate cancer treatment. Anticancer Drugs 16: 417-422, 2005.

28. Beatty GL and Paterson Y: Regulation of tumor growth by IFN-gamma in cancer immunotherapy. Immunol Res 24: 201-210, 2001.

29. Wu Y: New concepts of perioperative comprehensive therapy for non-small cell lung cancer. Zhongguo Fei Ai Za Zhi 8: 367-369, 2005 (In Chinese).

30. Chang JS, Chen LT, Shan YS, Lin SF, Hsiao SY, Tsai CR, Yu SJ and Tsai HJ: Comprehensive analysis of the incidence and survival patterns of lung cancer by histologies, including rare subtypes, in the era of molecular medicine and targeted therapy: A nation-wide cancer registry-based study from taiwan. Medicine (Baltimore) 94: e969, 2015.

31. Johnsen KB, Gudbergsson JM, Skov MN, Pilgaard L, Moos T and Duroux M: A comprehensive overview of exosomes as drug delivery vehicles-endogenous nanocarriers for targeted cancer therapy. Biochim Biophys Acta 1846: 75-87, 2014.

32. Zeller T, Muenstedt K, Stoll C, Schweder J, Senf B, Ruckhaeberle E, Becker S, Serve $\mathrm{H}$ and Huebner J: Potential interactions of complementary and alternative medicine with cancer therapy in outpatients with gynecological cancer in a comprehensive cancer center. J Cancer Res Clin Oncol 139: 357-365, 2013.

33. Puetzler J, Feldmann RE Jr, Brascher AK, Gerhardt A and Benrath J: Improvements in health-related quality of life by comprehensive cancer pain therapy: A pilot study with breast cancer outpatients under palliative chemotherapy. Onco Res Treat 37: 456-462, 2014.

34. Boss DS, Glen H, Beijnen JH, Keesen M, Morrison R, Tait B, Copalu W, Mazur A, Wanders J, O'Brien JP, et al: A phase I study of E7080, a multitargeted tyrosine kinase inhibitor, in patients with advanced solid tumours. Br J Cancer 106: 1598-1604, 2012.

35. Yamada K, Yamamoto N, Yamada Y, Nokihara H, Fujiwara Y, Hirata T, Koizumi F, Nishio K, Koyama N and Tamura T: Phase I dose-escalation study and biomarker analysis of E7080 in patients with advanced solid tumors. Clin Cancer Res 17: 2528-2537, 2011

36. Hainsworth JD, Spigel DR, Burris HA III, Waterhouse D, Clark BL and Whorf R: Phase II trial of bevacizumab and everolimus in patients with advanced renal cell carcinoma. J Clin Oncol 28: 2131-2136, 2010.

37. Harzstark AL, Small EJ, Weinberg VK, Sun J, Ryan CJ, Lin AM, Fong L, Brocks DR and Rosenberg JE: A phase 1 study of everolimus and sorafenib for metastatic clear cell renal cell carcinoma. Cancer 117: 4194-4200, 2011.

38. Lippitz BE: Cytokine patterns in patients with cancer: A systematic review. Lancet Oncol 14: e218-e228, 2013.

39. Whiteside TL: Inhibiting the inhibitors: Evaluating agents targeting cancer immunosuppression. Exp Opin Biol Ther 10: 1019-1035, 2010. 